\title{
Predictors of Vascular Calcification in Hemodialysis Patients
}

\section{Said Sayed Ahmed Khamis' ${ }^{1}$ Ahmed Ragheb Tawfeek ${ }^{1}$, Mohammad Gamal Al Din Al-Helbawy ${ }^{2}$, Mohammad EL-Sayed Abdel Samea ${ }^{3}$, Heba Kamal Abd El Khalek ${ }^{*}$}

\author{
${ }^{1}$ Internal Medicine Department, Faculty of Medicine, Menoufia University, Shibin El Kom, Egypt \\ ${ }^{2}$ Clinical Pathology Department, Faculty of Medicine, Menoufia University, Shibin El Kom, Egypt \\ ${ }^{3}$ Diagnostic Medical and Interventional Radiology Department, National Liver Institute, Menoufia University, \\ Shibin El Kom, Egypt \\ Email: *dr.hebakamal88@yahoo.com
}

How to cite this paper: Khamis, S.S.A., Tawfeek, A.R., Al Din Al-Helbawy, M.G., Samea, M.E.-S.A. and El Khalek, H.K.A. (2021) Predictors of Vascular Calcification in Hemodialysis Patients. Open Journal of Nephrology, 11, 273-280.

https://doi.org/10.4236/ojneph.2021.112022

Received: April 5, 2021

Accepted: June 19, 2021

Published: June 22, 2021

Copyright (๑) 2021 by author(s) and Scientific Research Publishing Inc. This work is licensed under the Creative Commons Attribution International License (CC BY 4.0).

http://creativecommons.org/licenses/by/4.0/

\section{(c) (i) Open Access}

\begin{abstract}
Background: The prevalence of both cardiovascular mortality and vascular calcification is much higher in patients with chronic kidney disease (CKD) than in the general population so early detection and intervention of VC may prevent or delay the progression and achieve improved patient outcomes. Objectives: To detect different predictors of vascular calcification in haemodialysis patients. Methods: This was a cross sectional observational study that included 85 patients with end stage renal disease (ESRD) on regular dialysis 47 males and 38 females ranged between 18 and 80 years old selected from Nephrology Unit, Internal Medicine Department, Menoufia University Hospital from April 2019 to May 2020. Serum calcium, phosphorus, alkhaline phosphatase, intact PTH, serum Matrix GLA protein, vitamin D and non-contrast CT for calcium scoring of femoral arteries were performed. Results: There was a significant correlation between age of the patient by years ( $p$ value 0.0001 ), serum calcium ( $\mathrm{p}$ value 0.0001 ), phosphate $(0.0001)$, calcium phosphate products ( $\mathrm{p}$ value 0.0001$)$ and alkhaline phosphatase (0.0001), and vascular calcification score detected by non-contrast $\mathrm{CT}$ on femoral arteries and negative correlation between serum Matrix GLA protein $p$ value 0.0001 ) and the detected calcification score; the lower the MGP level the higher calcification score while there was no correlation between body mass index (BMI) ( $\mathrm{p}$ value 0.021 ) intact PTH ( $\mathrm{p}$ value 0.117 ), serum vitamin $\mathrm{D}$ level ( $\mathrm{p}$ value 0.643 ), serum albumin ( $\mathrm{p}$ value 0.643 ), serum haemoglobin ( $\mathrm{p}$ value 0.257 ) and duration of dialysis ( $p$ value 0.260 ) and the detected score. Serum phosphate and calcium phosphorus product are independent risk factors for vascular calcification severity in haemodialysis patients. Conclusions: serum phosphate, calcium phosphate products are risk factors for vascular calcification while
\end{abstract}


intact PTH vitamin D has no significant role in developing vascular calcification in hemodialysis patients. Matrix GLA protein was inversely correlated with vascular calcification score.

\section{Keywords}

Vascular Calcification, Hemodialysis End Stage Renal Disease

\section{Introduction}

Chronic kidney disease patients are at a ten-fold higher risk of developing cardiovascular disease than age-matched controls. Being responsible for $54 \%$ of the deaths of ESRD patients [1].

Patients on dialysis experience, on average, a $15 \%$ annual increase in coronary calcification, likely a significant contributor to death [2].

Numerous risk factors for vascular calcification have been reported. Traditional risk factors include advanced age, hypertension, diabetes, and dyslipidemia. Non-traditional risk factors in CKD include disorders of mineral metabolism, elevated serum PTH levels, excessive intake of calcium supplements, inflammation, malnutrition, and oxidative stress [3].

Vascular calcification is not a passive process where dysregulation of calcium and phosphorus results in precipitation of minerals in the vessel walls as previously thought, but an active one, whereby cells actively deposit calcium in the vessel wall, akin to bone formation [4].

As kidney function declines, phosphate $\left(\mathrm{PO}_{4}\right)$ accumulates in serum and binds to calcium (Ca). Once the concentration of $\mathrm{Ca} / \mathrm{PO}_{4}$ particles in serum exceeds their saturation point, they precipitate and induce the vascular smooth muscle cells (VSMC) of the arteries to undergo differentiation into an osteogenic phenotype in which the VSMC calcify and stiffen [5].

Hyperphosphatemia induces VSMCs and endothelial cells to undergo apoptosis and to release matrix vesicles containing procalcific substances, increase in reactive oxygen species production and ultimately leading to calcification [6].

\section{Patient and Method}

This was a cross sectional observational study included 85 patients with end stage renal disease (ESRD) on regular dialysis. This study was carried out in Nephrology Unit, Internal Medicine Department, Menoufia University Hospital from April 2019 to May 2020. This study followed the ethical standards of our institute and approved by ethical committee of Menoufia University Hospital. All subjects were given informed consent. All patients were 18 years old on regular hemodialysis 4 hours three sessions/week. The following patients were excluded from the study: less than 18 years, patients with hemodialysis vintage less than 6 months and Patients with known malignancy. All patients underwent thorough history taking, clinical examination, laboratory investigations included 
calcium, phosphorus, Alkaline phosphatase, serum Matrix GLA protein, vitamin D level serum albumin, haemoglobin and intact $\mathrm{PTH}$, non-contrast computed tomography for calcium scoring of both right and left femoral arteries was done for all patients and the higher score of both femoral arteries was considered in our results.

Non-contrast CT femoral arteries was done to detect and quantify calcification through the volume of femoral arteries using the Somatom definition 128 slices, Siemens healthiness, Erlangen, Germany. There were no current established dedicated protocols to calculate the femoral calcification score and hence, the syngo coronary calcium scoring software was utilized to quantify the calcium score that was also expressed as Agatston score [7].

\section{Statistical Analysis}

Data were collected and analysed using Statistical Package for Social Sciences program (SPSS) for Windows version 22.0 (SPSS Inc., Chicago, IL, USA) and Microsoft excel sheet. Numerical data were expressed as mean and standard deviation (SD) and Categorical data were presented as number and percentage. Spearman correlation was done to detect correlation between different variables and femoral artery calcification score. Univariate linear logistic regression analysis was done to detect independent predictor of vascular calcification. Receiving operation characteristic (ROC) curve were constructed to detect the cut off value of the vascular calcification predictors. $P$ value $<0.05$ was considered statistically significant.

\section{Results}

This is a cross sectional observational study included 85 end stage renal disease patients on regular hemodialysis. Vascular calcification was assessed using non contrast CT on femoral arteries.

Demographic, clinical and laboratory data of studied patients were shown in Table 1. Correlation between different clinical and laboratory data and vascular calcification score of femoral arteries by non-contrast CT are showen in Table 2. Univariate logistic regression analysis demonstrated that serum phosphate and calcium phosphorus product were independently predict occurrence of femoral vascular calcification. Receiving operation characteristic curve demonstrated that cut off point of serum phosphate was 5.45 and that of calcium phosphorus product was 47.05 with good AUC, specificity and sensitivity $(72.5 \%$ \& $85.3 \%)$ respectively for phosphate and $(74.5 \% \& 82.4 \%)$ respectively for calcium phosphorus product (Figure 1 and Table 3 ).

\section{Discussion}

Vascular calcification is common and associated with unfavorable outcomes among patients with end-stage renal disease (ESRD). Nevertheless, little is known whether the progression of vascular calcification outweighs the baseline calcification in association with overall and cardiovascular (CV) mortality in hemodialysis (HD) patients [8]. 
Table 1. Demographics, clinical and laboratory data of studied patients.

\begin{tabular}{|c|c|c|c|}
\hline Variable & $\mathrm{No}+\%$ & Range & Mean $\pm S D$ \\
\hline \multicolumn{4}{|l|}{ Gender } \\
\hline Male N (\%) & $42(49.4 \%)$ & & \\
\hline Female N (\%) & $43(50.58 \%)$ & & \\
\hline \multicolumn{4}{|l|}{ Diabetes } \\
\hline Yes N (\%) & $21(24.70 \%)$ & & \\
\hline No N (\%) & $64(75.29 \%)$ & & \\
\hline \multicolumn{4}{|l|}{ Hypertension } \\
\hline Yes N (\%) & $37(43.52 \%)$ & & \\
\hline No N (\%) & $48(56.47 \%)$ & & \\
\hline \multicolumn{4}{|l|}{ IHD } \\
\hline Yes N (\%) & $5(5.88 \%)$ & & \\
\hline No N (\%) & $80(94.11 \%)$ & & \\
\hline \multicolumn{4}{|l|}{ Heart failure } \\
\hline Yes N (\%) & $7(8.23 \%)$ & & \\
\hline No N (\%) & $78(91.76 \%)$ & & \\
\hline Age (years) & & $18-80$ & $(49.2824 \pm 15.513)$ \\
\hline Duration of dialysis & & $(0.5-20)$ & $(6.67+4.13)$ \\
\hline BMI & & $17-40$ & $26.46+5.27$ \\
\hline Hemoglobin (g/dl) & & $5.3-13.80$ & $9.80+1.52$ \\
\hline Calcium (mg/dl) & & $6.5-9.90$ & $8.68+0.62$ \\
\hline Phosphate (mg/dl) & & $3.20-8.30$ & $5.24+1.20$ \\
\hline Calcium phosphate product & & $26.27-76.30$ & $45.19+11.37$ \\
\hline Femoral score & & $0.00-2145$ & $81.54+310.42$ \\
\hline $\operatorname{ALP}(\mathrm{IU} / \mathrm{L})$ & & $26-506$ & $204+101.96$ \\
\hline Albumin (g/l) & & $2.30-4.90$ & $3.74+0.47$ \\
\hline MGP ng/ml & & $0.17-15.20$ & $1.46+2.02$ \\
\hline Vitamin D ng/ml & & $6.74+17.27$ & $12.005+2.65$ \\
\hline
\end{tabular}

Table 2. Correlation between femoral vascular calcification score and different clinical and laboratory parameters. That shows statistically significant correlation between detected femoral calcification score and serum calcium, phosphate, calcium phosphate product and alkaline phosphatase and MGP inversely correlated with calcification score.

\begin{tabular}{ccc}
\hline Variables & $\mathrm{R}$ & P value \\
\hline Age (years) & 0.609 & 0.0001 \\
Duration of dialysis (years) & 0.124 & 0.260 \\
BMI & 0.250 & 0.021 \\
Hemoglobin (g/dl) & 0.124 & 0.257 \\
Calcium (mg/dl) & 0.396 & 0.0001 \\
Phosphate (mg/dl) & 0.487 & 0.0001 \\
Calcium phosphate & 0.483 & 0.0001 \\
Intact PTH (pg/dl) & -0.171 & 0.117 \\
ALK IU & 0.436 & 0.0001 \\
Albumin (g/dl) & 0.049 & 0.654 \\
MGP & -0.531 & 0.0001 \\
Vitamin D & 0.087 & 0.643 \\
\hline
\end{tabular}


Table 3. Area under the curve (AUC), cut off value, sensitivity and specificity of phosphate and calcium phosphorus product for prediction of vascular calcification.

\begin{tabular}{cccccc}
\hline Variable & AUC & Cut of value & Specificity & sensitivity & P value \\
\hline CI $95 \%$ & 0.769 & 5.45 & $72.5 \%$ & $85.3 \%$ & 0.000 \\
Phosphate & $(0.665-0.874)$ & & & & \\
$\begin{array}{c}\text { Calcium phosphate } \\
\text { product }\end{array}$ & 0.778 & 47.05 & $74.5 \%$ & $82.4 \%$ & 0.000 \\
\hline
\end{tabular}

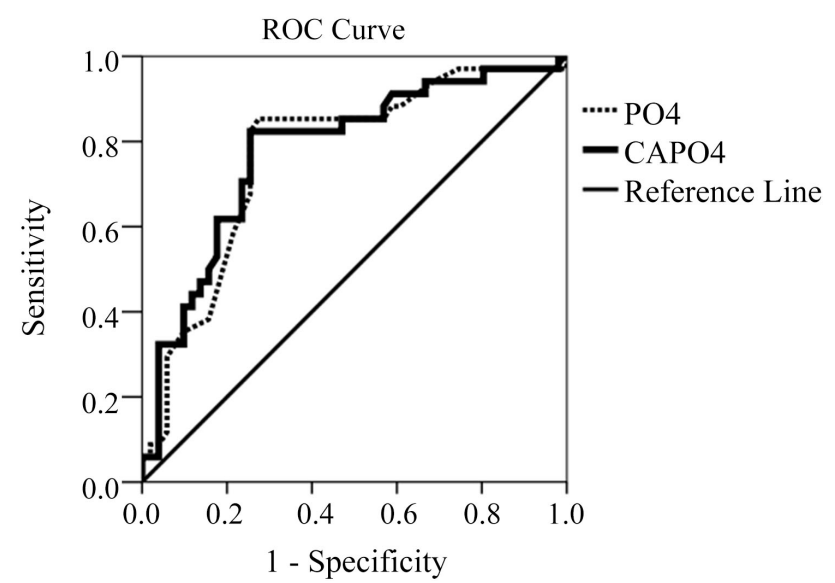

Figure 1. Receiving operation characteristic curve to detect area under the curve for phosphate and calcium phosphorus product in detecting vascular calcifications.

Our study included 85 patients on regular haemodialysis ranged between 18 and 80 years old.

Vascular calcification traditional risk factors include advanced age, hypertension, diabetes, and dyslipidemia. Non-traditional risk factors in CKD include disorders of mineral metabolism, elevated serum PTH levels, excessive intake of calcium supplements, inflammation, malnutrition, and oxidative stress. (3) In our current study age of the patient by years, serum calcium, serum phosphate, calcium phosphate product all have significant correlation with detected VC scores while serum phosphate and calcium phosphorus product in linear regression are independent risk factors for VC score. This agree with that Abnormal calcium and phosphate homeostasis is the main reason for stimulating VSMC calcification in patients with CKD [9] [10] [11] and with that Carotid intima media thickness has significantly positive correlation with traditional atherosclerotic risk factors like age, body mass index in CKD patients [12] and that age, BMI, phosphate, dialysis adequacy were associated with CAC progression [13] and the results of multivariate analysis indicated that only age and serum phosphate level were independent risk factors. VC is a serious complication of CKD and the prevalence was high. Some factors such as physical inactivity, history of IHD, dyslipidemia, older age, longer dialysis vintage, and lower levels of dialysate calcium concentration may contribute to VC in dialysis population [14]. 
In our results, there were no correlation between detected calcification score and duration of dialysis, body mass index, serum intact PTH, serum albumin and haemoglobin level and vitamin $\mathrm{D}$ level this disagree with that the mechanism of vascular calcification is multifactorial, and the active process of calcification advances along with dialysis duration [15]. As regard correlation between serum albumin and vascular calcification this disagree with that Studies on CAC have reported that the level serum albumin is inversely associated with CAC severity, [16] regarding to intact PTH this agree with that PTH cannot induce VC but have a synergistic effect with phosphate, probably because of an indirect association with bone remodeling and osteoclastic activity with a damaging result [17] and regarding to vitamin $\mathrm{D}$ our result agrees with thathttps://www.healio.com/authors/mwebb no changes in vascular calcification in patients with vitamin D insufficiency who were treated with cholecalciferol, though increases were seen in the placebo group. As kidney function declines, phosphate $\left(\mathrm{PO}_{4}\right)$ accumulates in serum and binds to calcium (Ca) [18].

There was negative significant correlation between serum MGP and the detectedVC score; the lower MGP level the more calcification score this agree with that Matrix Gla protein is recognized as a potent local inhibitor of vascular calcification. There are some limitations of our work; the study was observational without any intervention to detect the beneficial effect of controlling vascular calcification progression risk factors [19]-[24].

\section{Conclusions}

High serum phosphate and calcium phosphate product levels in hemodialysis patients are the main predictors and risk factors for vascular calcification. Neither vitamin D nor intact PTH has correlation with detected vascular calcification score. MGP was inversely correlated with vascular calcification score. This study has the limitation of having small sample size and it was cross sectional observational study.

Recommendation: a multi-center prospective randomized control trial with interventional controlling of vascular calcification risk factors is required to detect the beneficial effect of controlling these risk factors on progression of vascular calcification. Conflicts of Interest: The authors declare no conflicts of interest regarding the publication of this paper.

\section{Conflicts of Interest}

The authors declare no conflicts of interest regarding the publication of this paper.

\section{References}

[1] Saran, R., Li, Y. and Robinson, B. (2014) US Renal Data System 2015 Annual Data Report: Epidemiology of Kidney Disease in the United States. American Journal of Kidney Diseases, 67, A7-A8.

[2] Daniel, W.T., Weber, C., Bailey, J.A., Raggi, P. and Sharma, J. (2013) Prospective 
Analysis of Coronary Calcium in Patients on Dialysis Undergoing a Near-Total Para Thyroidectomy. Surgery, 154, 1315-1321; discussion 21-22. https://doi.org/10.1016/j.surg.2013.06.030

[3] Raggi, P. (2017) Cardiovascular Disease: Coronary Artery Calcification Predicts Risk of CVD in Patients with CKD. Nature Reviews Nephrology, 13, 324-326. https://doi.org/10.1038/nrneph.2017.61

[4] O’Neill, W.C. (2007) Vascular Calcification: Not So Crystal Clear. Kidney International, 71, 282-283. https://doi.org/10.1038/sj.ki.5002119

[5] Yamada, S. and Giachelli, C. (2017) Vascular Calcification in CKD-MBD: Roles for Phosphate, FGF23, and Klotho. Bone, 100, 87-93. https://doi.org/10.1016/j.bone.2016.11.012

[6] Gross, P., Six, I., Kamel, S. and Massy, Z.A. (2014) Vascular Toxicity of Phosphate in Chronic Kidney Disease: Beyond Vascular Calcification. Circulation Journal, 78, 2339-2346. https://doi.org/10.1253/circj.CJ-14-0735

[7] Agatston, A.S., Janowitz, W.R., Hildner, F.J., Zusmer, N.R., Viamonte Jr., M. and Detrano, R. (1990) Quantification of Coronary Artery Calcium Using Ultrafast Computed Tomography. Journal of the American College of Cardiology, 15, 827-832. https://doi.org/10.1016/0735-1097(90)90282-T

[8] Chung, W.-S., Shih, M.-C., Wu, P.-Y., Huang, J.-C., Chen, S.-C., Chiu, Y.-W., Chang, J.-M. and Chen, H.-C. (2020) Progression of Aortic Arch Calcification Is Associated with Overall and Cardiovascular Mortality in Hemodialysis. Disease Markers, 2020, Article ID: 6293185. https://doi.org/10.1155/2020/6293185

[9] Lu, K.C., Wu, C.C., Yen, J.F. and Liu, W.C. (2014) Vascular Calcification and Renal Bone Disorders. The Scientific World Journal, 2014, Article ID: 637065. https://doi.org/10.1155/2014/637065

[10] Houben, E., Neradova, A., Schurgers, L.J. and Vervloet, M. (2016) The Influence of Phosphate, Calcium and Magnesium on Matrix Gla-Protein and Vascular Calcification: A Systematic Review. Giornale Italiano di Nefrologia, 33, gin/33.6.5.

[11] Moe, S.M. and Chen, N.X. (2008) Mechanisms of Vascular Calcification in Chronic Kidney Disease. Journal of the American Society of Nephrology, 19, 213-216. https://doi.org/10.1681/ASN.2007080854

[12] Reddy, S., Kumar, P., Mastan Valli, B. and Shaik, M.V. (2015) Carotid Artery Disease in Patients with Chronic Kidney Disease. Journal of Medical Science and Clinical Research, 3, 5260-5271.

[13] Shang, D., Xie, Q., Ge, X., Yan, H., Tian, J., Kuang, D., Hao, C.M. and Zhu, T. (2015) Hyperphosphatemia as an Independent Risk Factor for Coronary Artery Calcification Progression in Peritoneal Dialysis Patients. BMC Nephrology, 16, Article No. 107. https://doi.org/10.1186/s12882-015-0103-8

[14] AlGhonaim, M.A. and Fathalla, A.A. (2019) Vascular Calcification in Patients with Chronic Kidney Disease on Dialysis in the Kingdom of Saudi Arabia: A Cross-Sectional Study. Saudi Journal of Kidney Diseases and Transplantation, 30, 571-580. https://doi.org/10.4103/1319-2442.261329

[15] Ohtake, T. and Kobayashi, S. (2017) Impact of Vascular Calcification on Cardiovascular Mortality in Hemodialysis Patients: Clinical Significance, Mechanisms and Possible Strategies for Treatment. Renal Replacement Therapy, 3, Article No. 13. https://doi.org/10.1186/s41100-017-0094-y

[16] Ohtake, T., Ishioka, K., Honda, K., Oka, M., Maesato, K., Mano, T., et al. (2010) Impact of Coronary Artery Calcification in Hemodialysis Patients: Risk Factors and Associations with Prognosis. Hemodialysis International, 14, 218-225. 
https://doi.org/10.1111/j.1542-4758.2009.00423.x

[17] Graciolli, F.G., Neves, K.R., dos Reis, L.M., Graciolli, R.G., Noronha, I.L., Moysés, R.M. and Jorgetti, V. (2009) Phosphorus Overload and PTH Induce Aortic Expression of Runx2 in Experimental Uraemia. Nephrology Dialysis Transplantation, 24, 1416-1421. https://doi.org/10.1093/ndt/gfn686

[18] Webb, M.J. (2020) FDA Approves First Treatment for Primary Hyperoxaluria Type 1. Healio News, 4 August.

[19] Puzantian, H., Akers, S.R., Oldland, G., Javaid, K., Miller, R., Ge, Y., Ansari, B., Lee, J., Suri, A., Hasmath, Z. and Townsend, R. (2018) Circulating Dephospho-Uncarboxylated Matrix Gla-Protein Is Associated with Kidney Dysfunction and Arterial Stiffness. American Journal of Hypertension, 31, 988-989. https://doi.org/10.1093/ajh/hpy079

[20] Jaminon, A.M., Dai, L., Qureshi, A.R., Evenepoel, P., Ripsweden, J., Söderberg, M., Witasp, A., Olauson, H., Schurgers, L.J. and Stenvinkel, P. (2020) Matrix Gla Protein Is an Independent Predictor of Both Intimal and Medial Vascular Calcification in Chronic Kidney Disease. Scientific Reports, 10, Article No. 6586. https://doi.org/10.1038/s41598-020-63013-8

[21] Ziegler, S.G., Gahl, W.A. and Ferreira, C.R. (2018) Chapter 32. Disorders and Mechanisms of Ectopic Calcification. In: Genetics of Bone Biology and Skeletal Disease, 2nd Edition, Academic Press, 571-595. https://doi.org/10.1016/B978-0-12-804182-6.00032-0

[22] Chiyoya, M., Seya, K., Yu, Z., Daitoku, K., Motomura, S., Imaizumi, T., Fukuda, I. and Furukawa, K.I. (2018) Matrix Gla Protein Negatively Regulates Calcification of Human Aortic Valve Interstitial Cells Isolated from Calcified Aortic Valves. Journal of Pharmacological Sciences, 136, 257-265. https://doi.org/10.1016/j.jphs.2018.03.004

[23] Bjørklund, G., Svanberg, E., Dadar, M., Card, D.J., Chirumbolo, S., Harrington, D.J. and Aaseth, J. (2020) The Role of Matrix Gla Protein (MGP) in Vascular Calcification. Current Medicinal Chemistry, 27, 1647-1660. https://doi.org/10.2174/0929867325666180716104159

[24] Barrett, H., O’Keeffe, M., Kavanagh, E., Walsh, M. and O'Connor, E.M. (2018) Is Matrix Gla Protein Associated with Vascular Calcification? A Systematic Review. Nutrients, 10, 415. https://doi.org/10.3390/nu10040415 